\title{
Entre los intersticios de la democracia: Las revistas estudiantiles, la universidad uruguaya en transición y las pujas políticas por los significados de la democracia
}

\author{
Between the interstices of democracy: \\ Student magazines, the Uruguayan university in transition \\ and contestations over the meaning of democracy
}

https://doi.org/10.22235/d33.2376

Gabriela González Vaillant

ORCID: 0000-0002-7970-4195

Universidad Católica del Uruguay / Universidad de la República

RESUMEN*

El presente trabajo analiza el proceso de reorganización del movimiento estudiantil universitario uruguayo en los primeros años de la década de los ochenta y especialmente en periodo comprendido entre 1980 y 1983 . El trabajo busca rastrear la génesis de la (re)constitución de este actor social a través de una mirada atenta a las revistas estudiantiles, proponiendo una mirada alternativa a la transición democática más allá de lo político partidario. El trabajo concluye que las revistas estudiantiles constituyeron canales alternativos de expresión, pero también espacios privilegiados para forjar y catalizar una identidad y sensibilidad generacional y estudiantil determinada durante un período en que no se encontraban disponibles canales tradicionales de participación y militancia.

Palabras clave: estudiantes; universidad; revistas; medios alternativos; Uruguay.

\begin{abstract}
This paper analyzes the process of reorganization of the Uruguayan university student movement in the early years of the eighties and specifically during the period between 1980 and 1983. The work seeks to trace the genesis of the reconstitution of this social and political actor to through a careful analysis of the student agazines that emerged during this period, proposing an alternative look at democratic transitions beyond the political partisan spheres. The work concludes that the student magazines constituted alternative channels of expression, but also privileged spaces to forge and catalyze a generational and student identity and sensitivity during a period in which traditional channels of participation and activism were unavailable.
\end{abstract}

Keywords: students; university; magazines; alternative media; Uruguay.

\footnotetext{
*Este artículo fue publicado originalmente en 2018, en el volumen 22(2) de la Revista de Historia Social y de las Mentalidades, editada por el Departamento de Historia de la Facultad de Humanidades de la Universidad de Santiago de Chile
}

(ISSN: 0717-5248 ISSN en línea: 0719-4749).

Enlace al artículo original: http://www.revistas.usach.cl/ojs/index.php/historiasocial/article/view/3643/26002939 


\section{Introducción}

El presente trabajo analiza el proceso de reorganización del movimiento estudiantil universitario uruguayo en los primeros años de la década de los ochenta y especialmente en periodo comprendido entre 1980 y 1983, año este último en que el movimiento estudiantil irrumpió en la escena pública con gran visibilidad y masividad. El trabajo busca rastrear la génesis de la (re)constitución de este actor social a través de una mirada atenta a las revistas estudiantiles que pulularon en el período en todas las facultades de la Universidad de la República. Propone mirarlas como canales alternativos de expresión, pero también como espacios privilegiados para forjar y catalizar una identidad y sensibilidad generacional y estudiantil determinada durante un período en que no se encontraban disponibles canales tradicionales de participación y militancia.

El siguiente fragmento de un cuento apareció en la revista estudiantil Diálogo a fines de 1982 sirve para dar cuenta, a través de la experiencia de un tal Juan, un estudiante de 18 años recién egresado a la universidad, del surgimiento de una nueva sensibilidad generacional en el contexto de la restauración democrática en Uruguay:

¡Novedades! ¡El año 80 es rico de ellas! Autos japoneses, sombrillas de Taiwán, los alquileres y las cuotas que suben y suben, pero también pasan otras cosas: la gente empieza hablar y algunos lo hacen hasta en voz alta (algo que Juan no recordaba haber visto). El Plebiscito está próximo y Juan escucha a su viejo opinar y discutir como antes, hasta lo ve más animado. El mismo se sorprende discutiendo con sus amigos, en el boliche, en el ómnibus. Llegado el 30 de noviembre, a Juan le hubiera gustado votar, y envidió a sus compañeros mayores; lo hubiera hecho por el "NO", como la mayoría de los muchachos que conocía. Juan y sus amigos se descubrieron diferentes a sus mayores: no tenía miedo, o tal vez no lo experimentaba en la forma intensa y real en que lo hacian sus padres. También se sintieron ingenuos, desinformados, aislados, manipulados, huérfanos de memoria histórica y de elementos para comprender su presente (ya el tiempo se había encargado de lavar la pintura de árboles y muros, y de robarse los colores, las caras, las consignas).

Hoy se habla de los partidos, de sus prohombres, de la tradición, de la transición. De la transacción; y se habla también del proceso, de la política económica, de la democracia, de la libertad. Juan y sus amigos se mueren por participar, pero se muerden los labios. No saben cómo actuar. Juan tiene mucha bronca contenida, quiere hacer algo por él y por los demás. Juan y sus amigos, ¿qué harán? (Revista “Juan y su generación", 1982, p. 34-35).

Los movimientos sociales constituyeron actores endémicos en los procesos de transición de la región (Garretón, 1989; Escobar y Alvarez, 1992). En lugar de ver a los movimientos sociales como actores esporádicos en el proceso de transición, este trabajo pretende poner de relieve cómo la transición también acontece en otros campos además del político-partidario y cómo un análisis exhaustivo de esos ámbitos puede, a su vez, ayudar a comprender los procesos macro políticos (de Giorgi y Demasi, 2015). Un estudio sobre los canales de comunicación estudiantiles durante los inicios de una década en transición (1980-1983) permitirá analizar cómo las profundas transformaciones sociales que acontecían a nivel macro y meso (de Giorgi y Demasi, 2015; Demasi, 2005; Rilla, 1997; Filgueira, 1985) impactaron al movimiento estudiantil y cómo, a su vez, los estudiantes contribuyen a dar forma a los acontecimientos que tuvieron lugar durante el período bajo estudio. Los procesos transicionales son momentos privilegiados y bisagra para muchos actores colectivos que tienen que repensarse a sí mismos luego de un hiato temporal frente a nuevos contextos donde la democracia y el valor de la libertad de expresión cobra otro sentido. En el caso de varios movimientos sociales, como veremos en el caso estudiantil, la transición a la democracia vino acompañada de una revalorización de la democracia 
participativa. Sin lugar a duda, la intrincada relación entre medios de comunicación y el poder político en los procesos de transición a la democracia ha sido objeto de análisis y debate; las demandas de democratización política siempre tuvieron sus corolarios en demandas por mayor libertad de expresión y comunicación (Bresnahan, 2007; Lugo-Ocando, 2008). Aunque el foco en general ha sido puesto en los medios de comunicación masivos, los medios alternativos de comunicación (como fueron las revistas estudiantiles, las radios y periódicos comunitarios) también jugaron un rol central en los procesos transicionales y en la difusión de un discurso contrahegemónico (Bresnahan, 1997; Wiley 2006; Buchanan, 1997).

Por otro lado, se pretende analizar la reconfiguración del movimiento estudiantil en tanto movimiento social a través de tres procesos claves que, aunque progresivos, son también mutuamente interdependientes y relacionados. Si bien los movimientos sociales muchas veces cobran visibilidad al irrumpir en la escena pública, la cara más visible, dichos procesos son siempre la punta de un iceberg y el reflejo de cierta capacidad de organización colectiva que se forja gracias a un trabajo identitario previo. El movimiento estudiantil uruguayo que irrumpe con fuerza en escena pública en 1983 no nace como un clavel del aire. Cuando el movimiento estudiantil uruguayo organiza la Semana del Estudiante en setiembre de 1983 (una serie de actividades estudiantiles que culminaron con una multitudinaria marcha en rechazo a la dictadura), ya era un actor social constituido, con capacidad de movilización, una idea compartida de demandas y reclamos, y cierta identidad común que aglutinaba a sus miembros en torno a la categoría inclusiva de "estudiantes" (aunque esta categoría esconda más de lo que esclarece). Es importante reflexionar y analizar los mecanismos por los cuales los estudiantes fueron transitando entre los intersticios de un régimen político en transición para irse constituyendo en tanto actor político colectivo. ¿Cómo se (re)constituyeron como movimiento estudiantil? ¿Qué papel jugaron las revistas en el proceso de construcción de una identidad compartida? ¿Quiénes constituyeron el "nosotros" y el "ellos" en ese proceso?
¿Cuáles eran y cómo se expresaron las demandas de ese movimiento en proceso de construcción?

En las ciencias políticas estadounidenses el debate de las "tres caras del poder" refiere a una controversia que surgió en los setenta en torno a un artículo de Robert Dahl (1957). Aunque no corresponde detenerse aquí en los pormenores de dicho debate, el mismo tiene implicancias importantes para comprender cómo operan los movimientos sociales y su capacidad de injerencia en la sociedad. En la formulación original de Dahl se proponía una definición conductual del poder inspirada en la idea de Weber: alguien tiene poder cuando logra que otro haga aquello que no haría por sí mismo. Frente a esta visión unidimensional de poder salieron al cruce Bachrach y Baratz (1962) proponiendo una definición bidimensional que introducía en el debate las fuerzas invisibles y latentes que constriñen lo que entra (y queda afuera) de la agenda pública. Mientras la definición más clásica de Dahl permitió iluminar la cara visible de los procesos de acción e interacción, esta segunda acepción puso en el tapete las fuerzas estructurales que habilitan (o inhabilitan) determinados tipos de discursos dentro de la opinión pública. Relacionado con esta acepción aparece la idea de "sesgo de movilización" y "ventana de oportunidades políticas" dentro de la literatura de movimientos sociales. Si bien este pasaje permitió pasar de miradas más manifiestas a otras más latentes de las fuerzas que determinan la interacción y el conflicto social, para Lukes (2005), el tercero en entrar al debate, ambas acepciones ignoraban una "tercera cara” del poder, que es aquella que opera en las percepciones, las preferencias, las comprensiones individuales y colectivas que hacen los sujetos de las situaciones. Este autor retoma la noción de hegemonía gramsciana e invita a considerar el conjunto de fuerzas operantes que hacen que los actores no puedan siquiera visualizar alternativas plausibles frente a un estado de situación dada. Esta tercera acepción permite analizar las fuerzas sociales y las prácticas institucionales que hacen del conflicto y la resistencia algo impensable en primera instancia.

Planteamos que en momentos cuando los canales de participación y protesta están bloqueados, el poder de 


\begin{tabular}{|c|c|c|}
\hline \multicolumn{2}{|c|}{ Cuadro 1. Dimensiones para estudiar la constitución de movimientos sociales } \\
\hline Cara de poder & Resultados & $\begin{array}{c}\text { Dimensión de constitución } \\
\text { del movimiento }\end{array}$ \\
\hline $\begin{array}{c}\text { Tercera cara } \\
\text { Latente }\end{array}$ & $\begin{array}{c}\text { ¿Quiénes somos? } \\
\text { Problematizar una situación, } \\
\text { concebirse como un actor colectivo } \\
\text { e identificar una solución determinada }\end{array}$ & Demarcaciones identitarias \\
\cline { 3 - 3 } $\begin{array}{c}\text { Segunda cara } \\
\text { Invisible }\end{array}$ & $\begin{array}{c}\text { iQué queremos? } \\
\text { Visibilizar la agenda de demandas } \\
\text { y obtener apoyo público } \\
\text { y de otros actores }\end{array}$ & Alianzas y foco de demanda \\
\cline { 3 - 3 } & $\begin{array}{c}\text { iCómo lo obtenemos? } \\
\text { Movilización de recursos } \\
\text { y protesta social }\end{array}$ & Marcos de demandas \\
\cline { 3 - 3 } Primera cara & Tácticas de protesta \\
\hline Visible & & Repertorios de protesta \\
\hline
\end{tabular}

Fuente: Creación propia en base a González Vaillant (2015)

los movimientos sociales, y en este caso el estudiantil, se juega en los intersticios de las dos caras de poder más invisibles enunciadas arriba. Los movimientos sociales pueden ejercer poder de movilización justamente cuando logran, en primer lugar, generar (en la mente, en los corazones) entre sus integrantes un conjunto de ideas acerca de problemáticas comunes que los aquejan (tercera cara del poder) y, en segundo lugar, a través de la construcción y difusión de un discurso de alternativas posibles (segunda cara del poder). La capacidad de movilización y protesta que se visibilizó en 1983 (primera cara del poder), solo fue posible gracias al trabajo de reconstrucción que aconteció previamente y durante el cual los medios de comunicación alternativos jugaron un papel central. Las revistas estudiantiles funcionaron en dicho contexto como catalizadoras de un sentir colectivo especifico, le dieron visibilidad, forjaron una identidad común, a la vez que le permitieron crecer, generar cierta estructura organizativa e integrar más estudiantes a sus filas. La nota editorial del primer número de la revista estudiantil Causa (Facultad de Derecho y Ciencias Sociales) planteaba de forma algo profética, el papel que irían a jugar estas revistas: "hemos dado un impulso, el primero, pero es sólo el comienzo, existe apenas el proyecto de un camino. Falta lo más importante y difícil, construirlo y caminar. Es un camino a recorrer a través de un medio de comunicación. Se trata de hacer sentir nuestra voz, de servir como medio de expresión de un sector de la sociedad, de un grupo de la sociedad que tiene intereses comunes y permanentes" ("Una causa que es nuestra", 1982). La tabla que sigue a continuación sintetiza las distintas dimensiones de construcción de un movimiento social, los resultados de dichos procesos y la cara de poder donde queda de manifiesto. En las páginas que siguen veremos cómo se fueron manifestando estas caras del movimiento estudiantil en la transición uruguaya.

Como toda cronología, la periodización de 1980-1983 es una construcción del investigador. La fecha inicial de la periodización se basa en la fundamentación de una serie de análisis politológicos e historiográficos (González, 1984; Caetano y Rilla, 1987; Rilla, 1997) que generalmente señalan este año como punto de partida de la transición en Uruguay, con el plebiscito en contra el proyecto constitucional de la dictadura cívico-militar y el triunfo de la oposición a la propuesta del gobierno y en el cual los estudiantes jugaron un papel fundamental (Demasi, 2005). Esta etapa transicional implicó una liberalización del régimen dictatorial y una reaparición de los partidos y de la sociedad civil en la escena política (González, 1985). El momento del retorno a la democracia se encontró con un auge históricamente novedoso de los movimientos sociales. El vacío relativo dejado por la proscripción de los partidos políticos abrió las posibilidades a que la sociedad uruguaya, acostumbrada al rol protagónico de los partidos, encontrara otros canales de expresión política colectiva. Es lo que se ha dado en llamar el momento "neocontractual" de la transición, donde el sistema político en momento democrático operó con una apertura a la negociación entre actores no mediatizados por los partidos políticos (Bayce, 1989). 
Ya cuando se crea la Asociación Social y Cultural de Estudiantes de Enseñanza Pública (ASCEEP ${ }^{1}$ ) en 1982, una sociedad civil que buscó llenar el vacío existente desde 1973 cuando la Federación de Estudiantes Universitarios del Uruguay (FEUU) fue disuelta como "asociación ilícita”, es posible hablar de un movimiento estudiantil constituido que no hace más que crecer. Ello le permite organizar actividades multitudinarias en torno a la Semana del Estudiante (17-25 de septiembre 1983), que culmina con una marcha y un acto en el Estadio Franzini el 25 de setiembre de 1983, y luego el Año Internacional de la Juventud 1984. Sin embargo, esa reconstitución también fue posible gracias a un entretejido de espacios de socialización informales e importantes (como ser asados, bienvenidas a nuevas generaciones, actividades deportivas) que- aunque a primera vista pueden parecer ajenas a las reivindicaciones políticas-cumplieron la función de ir gestando una identidad colectiva y generacional que fue clave para posteriores movilizaciones ${ }^{2}$. Dentro de este entramado de espacios de socialización y reconfiguración del tejido social, entran las revistas estudiantiles. En este sentido, en el Manifiesto por una Enseñanza Democrática leído el 25 de setiembre de 1983 en el mencionado acto, los estudiantes reconocen el papel fundamental de estos espacios en su reconstrucción:

Debemos destacar también la importancia que tuvo para todo el triunfo opositor en el plebiscito de 1980 en el cual la juventud jugó un rol determinante, es en este contexto y recogiendo las anteriores experiencias de movilización que se van generando nuevas formas organizativas y de expresión como son nuestros asados multitudinarios, nuestras murgas y nuestras revistas. Día a día se van generando nuevos espacios llegándose a la formación de una mesa interrevista y luego al fortalecimiento de ASCEEP, en este fortalecimiento de la Asociación inciden varios factores (Asociación Social y Cultural de Estudiantes de la Educación Pública, 1983, p.6).
Esta nueva identidad no estuvo exenta de tensiones inter e intrageneracionales, ya que el movimiento estudiantil que surge con la restauración democrática lo hace sobre un legado histórico determinado, pero en un contexto nuevo en el que emergieron nuevas sensibilidades e improntas generacionales (Araujo y Del Singore et. al., 1991). En tal sentido, la noción de democracia heredada de otras generaciones anteriores jugó un papel central en la configuración identitaria de estos jóvenes, ya sea por asimilación o por contraposición.

\section{Métodos y fuentes}

Este trabajo toma como punto de partida una combinación de testimonios, entrevistas en profundidad, estudio de documentos, textos y fuentes primarias disponibles en el Archivo General de la Universidad de la República y material de prensa del momento.

El Archivo General de la Universidad posee un rico y variado acervo de documentos y fuentes primarias donadas por muchos miembros de las generaciones que participaron activamente dentro del movimiento estudiantil en la década de 1980. Entre los materiales donados cabe destacar una predominancia de documentación perteneciente a la llamada generación de 1983 . Algunos documentos de interés fueron una serie de publicaciones periódicas estudiantiles como ser Diálogo, Conciencia, Estamos, Integrando, Catálisis, etc., rrecortes de periódicos, convocatorias y folletos para actividades estudiantiles, lo mismo que documentos y declaraciones del movimiento estudiantil.

En este trabajo se pone el foco en las revistas estudiantiles que se publicaron y distribuyeron previo a la

$1:$ : ASCEEP fue creada por estudiantes de Facultad de Derecho, dentro de la legalidad vigente, una sociedad civil sin fines de lucro con objetivos más o menos genéricos en sus inicios pero que logró ir generando un espacio de participación y organización.

2:: Según Markarian, Jung y Wschebor (2009) la ASCEEP pasó de 300 socios iniciales a 2.500 en 1983. 
Semana del Estudiante para analizar las formas en que se van gestando determinadas identidades generacionales dentro de los estudiantes, cómo se tematiza la relación con generaciones de estudiantes anteriores, la forma en que se describe el rol de los estudiantes en la sociedad y la forma en que se va construyendo una definición, más o menos compartida, más o menos controvertida, de la democracia.

Una nota sobre la forma. Se optó en general por incluir largos fragmentos de las citas analizadas para preservar el contexto original y, a su vez, dar voz a esos estudiantes en sus propias palabras.

Las revistas estudiantiles: esos medios alternativos de comunicación

Es posible identificar indicios de movilización estudiantil y resistencia clandestina previos a la irrupción estudiantil en el ochenta que dotaron de un legado organizacional y simbólico a las posteriores generaciones. Algunos eventos que cabe señalar fueron los sucesivos recordatorios de la muerte de Líber Arce a partir de 1968 (Sempol, 2004); los reclamos universitarios por demandas particulares, como ser la oposición a medidas que buscaron restringir el ingreso a la universidad, la creación de la mesa central de la Federación de Estudiantes Universitarios del Uruguay (FEUU) en 1978 sobre la base de las juventudes comunista y socialista (Porrini, 2012) y la reaparición de publicaciones periódicas estudiantiles hacia fines de los setenta (por ejemplo "Jornada" y "El Estudiante Libre") (ver Markarian, Jung y Wschebor, 2009). Por otra parte, entre 1978 y 1980 se realizan diversas actividades en varias Facultades que buscaron generar espacios de socialización y reconstrucción del tejido social a través de reivindicaciones de tipo curricular y académicas. Finalmente, en el contexto del plebiscito del 80, se promovieron un conjunto de acciones para ganar adhesiones para el NO, generándose un espacio de mayor activismo y fortaleciéndose un bloque de movilización anti-dictadura, aunque el mismo operó, en una primera instancia, en forma principalmente clandestina. Los testimonios acerca de las interacciones, las organizaciones y las solidaridades que se tejieron en estos procesos contradicen la tesis de ausencia de transición generacional ya que estos movimientos que operaron en la clandestinidad a través de grupos de base fueron buscando ganar espacios de legalidad.

En este contexto, en 1981 se produce la irrupción de revistas universitarias como forma de organización y difusión legal que buscaron abrir espacios de reflexión colectivas y la concomitante creación de la Coordinadora de Revistas.

En marzo de 1981 surgió la primera revista universitaria en la Facultad de Arquitectura (Trazo) y en octubre de ese mismo año surge Dialogo, que, aunque nació principalmente como una iniciativa de estudiantes independientes de Derecho, buscó nuclear a estudiantes universitarios de todas la Facultades y posteriormente también a estudiantes de secundaria. ${ }^{3}$ La revista Diálogo se constituyó en una publicación de carácter universitario, no oficial, elaborada por estudiantes con la aspiración de ser la voz del sector estudiantil "dentro y fuera de la universidad" para, en sus propias palabras en un simposio sobre los jóvenes y los medios de comunicación, "romper el hielo, en una generación que estaba dejando en la historia un prolongado silencio de casi ocho años: una universidad adormecida, esclerosada, incapaz de asumir su rol como creadora de cultura, y a la vez mostrando con mayor evidencia su carácter clasista por medio del polémico examen de ingreso" ("Diálogo: una experiencia de participación”, 1982, p. 1). Ya desde su surgimiento se vislumbran tensiones entre una identidad generacional específica, intereses estudiantiles particularistas y una identidad social en transición que buscó romper con el imaginario que la dictadura quiso instaurar de una juventud despolitizada. La revista Diálogo constituyó un primer intento por articular el trabajo entre las diferentes facultades de la Universidad, algo que había sido imposible hasta ese momento, y se convirtió por la vía de los

3:: La revista cesa de publicarse a mediados de 1983 en su $11^{\circ}$ número. 
hechos en un intento proto-gremial. Al decir de Jorge Rodríguez, quien fue el primer presidente de ASCEEP, "la revista Diálogo logró reunir un pequeñito grupo de redacción en cada facultad. Entonces ese consejo de redacción que se juntaba en el local o garaje que tenía por allá abajo era un espacio porto-gremial. Tenía toda la intención. Entonces ahí lo de diálogo abrió un camino, cuán hasta que salió la revista de facultades, salió Trazo, revista arquitectura, era una buena revista que tenía contenido académico y también tenía contenido crítico gremial con extremo cuidado. Y a partir de eso empezaron a salir en todos lados".

Entre mediados de 1981 y 1982, comenzaron a pulular las revistas universitarias en las diversas $\mathrm{Fa}-$ cultades (Balance en Ciencias Económicas, Catálisis de Química, Causa de Derecho y Ciencias Sociales, Encuentro Veterinario en Veterinaria, Integrando de Ingeniería, Salud de Medicina, Siembra en Agronomía) y concomitantemente se gestó la organización de espacios más orgánicos de participación (aunque aún de resistencia implícita), como fue la creación de la ASCEEP y el Club de Ingeniería. Este mismo año, se consolidó la "Mesa Inter revistas", que agrupó a distintos núcleos de las revistas y cuya actividad trascendieron las tareas periodísticas, y se pactó lanzar la Semana del Estudiante para setiembre del 83 (Calicchio, 2002). Al decir de Jung (2010), aunque en un comienzo el carácter opositor y de resistencia no se explicitó abiertamente, estas revistas constituyeron un espacio alternativo que permitió, por un lado, cohesionar y visibilizar al actor estudiantil e impulsar la participación juvenil durante estos años y, por el otro, constituirse en tanto "esfera pública alternativa" a los medios oficiales (Marchesi, 2009, p. 378).

Los medios alternativos de comunicación en los procesos de transición a la democracia jugaron un doble proceso; interno y externo. Más allá del impacto que tuvieron las publicaciones al interior del ámbito estudiantil, las mismas se constituyeron un canal de expresión que trascendió dicho ámbito, que comenzó a visibilizar al actor estudiantil y difundir sus perspectivas y que permitió comenzar a construir espacios comunicacionales contrahegemónicos. El semanario blanco $L a$ Democracia anunció la llegada de "una Revista Joven" en abril de 1982, "que busca llenar un vacío existente, desde hace mucho tiempo, en nuestro país (...) En anteriores números de nuestro semanario intentamos saludarlos, pero nos sorprendió la clausura. Vaya pues nuestro saludo y deseo que el diálogo sea fructífero" ("Una revista joven”, 1982, p. 5). Por su parte, la revista El Dedo en su segundo número en una caricatura titulada "Para No Pasar Vergüenza”, incluyó a la Revista Diálogo como un adminiculo imprescindible para todo asiduo asistente a un recital de canto popular (junto con el gamulan o campera "arquitectura look", la cara profundamente filosa y los anteojos finos), sugiriendo su lugar indiscutido dentro de la cultura juvenil alternativa de la época y mostrando como dichas publicaciones lograron trascender los espacios comunicacionales circunscriptos a los centros educativos.

Es interesante recalcar la creatividad que desplegaron muchas veces las revistas estudiantiles para criticar a la dictadura en un momento en que, si bien comenzó a evidenciarse mayor tolerancia a la disidencia, la censura y la represión aun eran moneda corriente. Es posible ver una progresiva politización de los editoriales y artículos; mientras los primeros números fueron en general cautelosos respecto al contenido y alcance, refiriendo genéricamente a "cuestiones estudiantiles", paulatinamente aparecen artículos abiertamente críticos. Por citar un ejemplo, en su primer año la Revista Diálogo refería al "estado actual" de las calles de Montevideo, utilizando nombres de emblemáticas calles de la ciudad para realizar una aguda critica a la situación institucional imperante en Uruguay durante la transición a la democracia.

CONSTITUCIÓN. Está bonita y antigua calle que tanto queremos los uruguayos. Se encuentra clausurada y a la espera de su próxima remodelación. Provisoriamente se la conoce con el nombre de ACTO INSTITUCIONAL. 
DEMOCRACIA. Se encuentra cerrada al igual que el anterior. De fuentes oficiales se anuncia su próxima reapertura para noviembre de este año, pero esta será solamente parcial, ya que se permitirá únicamente circular por la mano derecha. El nombre actual de esta añorada calle no lo pudimos ver, porque la chapa estaba borrosa.

LIBERTAD. Su estado es realmente calamitoso. Fundamentalmente el deterioro se hace viento en la cuadra denominada DE EXPRESIÓN, la que, debido a las reiteradas clausuras, es prácticamente imposible de transitar. Los lugareños reclaman en forma tenaz ensanchamiento de esta arteria, que es vital para volver a ARTIGAS.

DEFENSA. A diferencia de las anteriores nombradas, luce esplendorosa. Sin embargo, hay mucha gente que considera que los cuantiosos gastos que se realizan en ella son la causa del patético deterioro de ECONOMÍA, TRABAJO, PROGRESO y otras importantes calles ("El estado actual de nuestras calles”, 1982, p. 13).

Las revistas buscaron formas ingeniosas de ir marcando la oposición al régimen, en un primer momento publicando artículos que daban guiños a los lectores más atentos. Aunque las revistas del área social, por su propio objeto de estudio, realizaron críticas más evidentes, este proceso fue una constante en todas las publicaciones estudiantes de estos años. El editor responsable de la revista de la Facultad de Veterinaria, que jugó un papel protagónico en el movimiento estudiantil de la transición democrática, recuerda ese proceso de politización creciente del contenido de las revistas de la siguiente manera: "en el primer número eran todos artículos técnicos (...) Después el segundo número tenía artículos técnicos de veterinaria, pero habían otros de otros docentes, por ejemplo un docente que estaba exiliado en Costa Rica, que después fue decano post-in- tervención (...) Era un artículo publicado en una revista en Costa Rica llamado "La investigación integrada al proceso educativo", nada menos que eso (...). En el 82 sacamos un suplemento en contra del examen de admisión con recortes de prensa de la época" (Rubianes, E., comunicación personal, 27 de julio de 2018). El primer número de Encuentros Veterinarios en noviembre de 1981 definía como parte de su propósito ir "ocupado ese vacío que todos notamos y que parece tan necesario llenar" ("Editorial", 1981, p. 1); en julio de 1984 "trazar los imprescindibles caminos de participación para que a lucha por la Universidad Democrática y verdaderamente popular sea tarea de todos" (“Editorial", 1981, p. 1).

En octubre de 1982, se realizó un simposio "Los jóvenes y los medios de comunicación" organizado por el Foro Juvenil. Las revistas universitarias, invitadas a participar, realizaron una ponencia colectiva para expresar su punto de vista respecto al rol y la experiencia de las revistas universitarias hasta el momento. Su ponencia fue transcripta en la revista Causa en agosto de 1982. Las revistas estudiantiles se presentaron como un medio alternativo de comunicación frente a la prensa masiva, oponiéndose a los medios oficialistas empresariales, a los que se acusó de dejar al lector en papel de "simple espectador". Las revistas estudiantiles, por contraposición, se vieron a sí mismas como actores proactivos con estructuras abiertas y participativas que buscaron regenerar espacios de sociabilidad, a través de nuevas formas de expresión y comunicación. En propias palabras de los estudiantes en el mencionado simposio:

(...) analizándolas como medios de comunicación, las revistas tienen ciertas características que le son propias, y que se oponen a la de los tradicionales medios de comunicación. Éstos responden a una organización empresarial de tipo burocrático, conformando un oligopolio que deja al lector el papel de simple espectador. Establecen una serie de canales que, unilateralmente, llegan a invadir la vida privada, mediatizando 
las relaciones humanas. (...) Las revistas universitarias, en cambio, se identifican con el medio que las produce, ya que son estructuras propias de los estudiantes, que les devuelven su derecho a ser protagonistas de su historia. Son estructuras abiertas y profundamente participativas, que establecen una relación directa entre quienes las emplean como medio de comunicación. Cumplen con el fin de rendir un servicio a sus usuarios, buscando brindar una información jerarquizada de acuerdo a sus necesidades, y promoviendo a través de planteos y cuestionamientos, la formación de una opinión seriamente fundada. (...) Constituyen una alternativa que reivindica, por su existencia y funcionamiento, el derecho a la libre información y, sobre todo, el derecho de dirigir los medios de comunicación inherente a toda sociedad que levante la bandera de la democracia. ("La Juventud universitaria y los medios de comunicación”, 1982, p. 17).

Si bien un análisis de las revistas y sus contenidos permiten reconstruir la génesis de una articulación político-social, los archivos escritos en este caso no permiten dar cuenta de las instancias de negociación y mediatización de esta trama en relación con el proceso de construcción mismo de esos espacios. Las revistas, en tanto espacios públicos de interlocución, dieron la idea de espacios inherentemente democráticos, pero es claro que varios de sus integrantes tenían doble militancia y debían manejar estos espacios comunicacionales con cautela. Los testimonios orales de algunos de sus protagonistas permiten ilustrar algunos de los procesos que, tras bambalinas, informaron las líneas editoriales y los artículos que veían la luz finalmente en las revistas. Quienes buscaron muchas veces radicalizar o politizar las posturas editoriales eran aquellos jóvenes que no estaban participando en espacios clandestinos de actividad política y esa interrogante de "hasta dónde tirar" de la cuerda estuvo siempre latente.
Conflicto adentro del espacio legal prácticamente no existía. Estaba el espacio de las cartas que pasaba el filtro del consejo de redacción (...). El consejo de redacción de a la revista estaba integrado en general, por lo menos en el caso de Veterinaria, por gente con doble militancia y otra que eran locos que laburaban, porque trabajaban y tenían que estar en el consejo de redacción, aunque no estuvieran en la militancia clandestina (...). El conflicto se daba más con los que militaban en el marco de la estructura clandestina, la discusión sobre el papel de la revista y qué hacer. Y, por ejemplo, pasaba mucho, que los locos querían a veces tirar más para delante de la cuerda de lo que el grupo que estaba clandestino entendía que era políticamente conveniente para el momento. Entonces, ese era el tema (Rubianes, E., comunicación personal, 27 de julio de 2018).

Una generación que quiere ser escuchada y la revalorización de la democracia

Durante el proceso de democratización en el Uruguay se comienzan a vislumbrar un cambio en la cultura política y un proceso de "reconversión ideológica" que se vio claramente reflejada en el plano discursivo con una revalorización de la democracia y un paulatino "abandono" de la idea de la transformación revolucionaria (Caetano y Rilla, 1995; Mieres y Argones, 1989). Por otro lado, se comienzan a procesar choques generacionales entre las culturas políticas pre-transicionales y nuevas culturas emergentes. En la década del 80 es posible trazar la aparición en nuestro país de la llamada generación dionisíaca (Perelli y Rial, 1986; Bayce, 1989) que se caracteriza por poner el énfasis en la calidad de vida cotidiana, en la participación, en las nuevas formas de expresión culturales.

Las revistas están plagadas de referencias al nacimiento de "una nueva generación", que se buscó diferenciar de sus antecesoras y, en especial, separar de 
esa idea de "silenciamiento" que se le había impuesto. Las revistas aparecían como un vaso comunicante de dichas voces que por fin serían escuchadas, permitiendo moverse desde una identidad latente a una manifiesta en la opinión publica (segunda cara del poder). Este afán por deshacerse del estigma de la llamada "generación del silencio" es una constante en todas las publicaciones estudiantiles de la época y estuvo muy presente en el discurso estudiantil de la generación 83. "No somos la generación del silencio, quisieron que fuéramos la generación del silencio, pero como siempre, los autoritarismos fracasan" se proclamó en el Manifiesto por una Enseñanza Democrática leído en el acto del Franzini ("Manifiesto por una enseñanza democrática”, 1983, p. 11). El extracto que sigue a continuación del ya mencionado simposio sobre los jóvenes y los medios de comunicación, en el que participaron las revistas estudiantiles, da cuenta de la responsabilidad depositada en las revistas estudiantiles como canales legales para apoyar la lucha por la democracia (externa) pero a través de formas de organización también mucho más democráticas (interna) (Causa, 1982, p.18). Es decir, los espacios de las revistas se constituyeron en micro mundos a escala del tipo de sociedad que se buscaba construir $\mathrm{y}$, en dicho sentido, fueron espacios de socialización y de construcción identitaria sumamente relevantes para las subjetividades estudiantiles del momento. Las revistas estudiantiles sirvieron como un espacio que buscó reflejar en su funcionamiento interno el tipo de solidaridad y vinculo que pretendía construir fuera de las paredes universitarias. Incluso, es posible decir que estas nuevas formas de organización dieron lugar a espacios más horizontales y menos jerárquicos que los que había conocido tradicionalmente el movimiento estudiantil uruguayo. Frente a la falta de experiencia y recursos materiales y técnicos se buscaron formas novedosas de organización y gestión:

"El fenómeno de las revistas universitarias ha venido a quebrar la inmovilidad superficial que ocultaba la efervescencia estudiantil; introduciendo en este vacío la voz del sector juvenil que se eleva para desmentir su apodo de la generación del silencio: aportando una nueva de dinámica que permite el desarrollo progresivo de la corriente renovadora dentro de la universidad".

"Los comienzos fueron difíciles, por un lado, porque para dar los primeros pasos hubo que vencer el aislamiento en que nos encontrábamos. Hubo que afirmar la confianza en nuestra capacidad de hacer, desestabilizada por una trayectoria enmarcada en la pasividad. Hubo que enfrentar la falta de experiencia. Por otro lado, estaba el problema de las carencias de recursos materiales y técnicos, hay que agregar una falta de apoyo generalizada, en algunos casos agraviada por la posición de las autoridades. (...)

El sistema democrático adoptado por los grupos desde un comienzo y fue necesario, y al crecer estos, instrumentar nuevas formas de participación: se subdividió el trabajo en la creación de comisiones que tomaron a su cargo todos aquellos que se fueron integrando" ("La juventud universitaria y los medios de comunicación”, 1982, p. 18).

Como el fragmento anterior ilustra, las revistas estudiantiles, tal como las teorizaron los propios estudiantes del momento, permitieron ir generando una nueva modalidad de militancia más velada y sigilosa que tuvo por finalidad reconstruir el tejido social del estudiantado que había quedado desarticulado, y así generar una identidad estudiantil colectiva. Dentro de este esfuerzo sistemático por regenerar un entretejido de espacios de socialización informales pero importantes las revistas permitieron dar difusión y visibilidad a dichos espacios. Las revistas estudiantiles sirvieron para promocionar y masificar los ya mencionados asados, las cooperativas de apuntes, las bienvenidas a las nuevas generaciones, 
las murgas y bailes y las actividades deportivas. Aunque a primera vista estas actividades puedan parecer ajenas a las reivindicaciones políticas, la función de gestar una identidad colectiva y generacional fue, no obstante, clave para viabilizar las posteriores movilizaciones (Markarian, Jung y Wschebor, 2009), y pasar así de la segunda a la primera cara de poder. Por otro lado, dichas actividades fueron surgiendo como espacios propicios para reclutar estudiantes a las filas de la militancia, en una primera instancia clandestina y paulatinamente más abierta. En este sentido, es interesante la reflexión que se realiza desde el Boletín de Estudiantes de Ingeniería de este proceso de transformación que permitió catalizar a las revistas desde espacios de socialización política más informal a gérmenes de actividad gremial:

Hasta el año 81 la facultad de ingeniería era un frío recinto fuertemente vigilado donde no existían condiciones para actividad gremial, ni siquiera para formar grupales de vida estudiantil. Lentamente, y con el esfuerzo de muchos compañeros durante meses, los estudiantes fuimos abriendo espacios públicos, buscando formas de Integración, organizando actividades masivas y construyendo en definitiva el germen de una actividad gremial. De esos primeros encuentros y discusiones fue surgiendo a la conciencia colectiva de una enseñanza antidemocrática, autoritaria y excluyente, a la que empezamos a responder, también por múltiples vías, con nuestras reivindicaciones y propuestas (...).

Durante los 16 meses que nos organizamos en el Club, dimos un proceso en el que la estructura $\mathrm{y}$ el trabajo fue tomando cada vez más forma y contenido gremiales, y en el que articulamos a través de ASCEEP, nuestra lucha con el resto de los estudiantes y con todo el movimiento popular.

A fines del año pasado los estudiantes nucleados en el Club decidimos retomar el nombre del his- tórico gremio de los estudiantes de ingeniería, en intención de avanzar de esta forma en la conquista de la libre agremiación, y reafirmando con este nombre nuestra voluntad de ser continuadores de las generaciones de estudiantes que nos precedieron “Breve reseña”, 1984, p. 1).

Las revistas formaron parte de un intento general por reconstruir un espacio de participación legal que permitiera revalorizar la democracia y la participación juvenil. En dicho sentido, las revistas constituyeron para muchos estudiantes ávidos por involucrarse verdaderas escuelas de participación. Esto no significa que no hubieran diferencias dentro del estudiantado en relación a distintas concepciones sobre la transición y el rol de los estudiantes en dicho proceso; las hubo y las revistas, especialmente durante sus últimos números, sirvieron como un espacio para dirimir rispideces y tensiones que fueron surgiendo en el seno del movimiento estudiantil (por citar un ejemplo, varias revistas explicitaron la discusión interna acerca de la legitimidad o no de ASCEEP como representante de los estudiantes con la FEUU ilegalizada). En un contexto de proscripción de los partidos políticos e ilegalización y persecución de grupos que históricamente han tenido fuerte injerencia dentro de la política universitaria (como ser el partido comunista y socialista) y e un momento en que las identidades partidarias no podían ser puestas sobre la mesa, las revistas dieron lugar a una suerte de "primavera" estudiantil donde las semejanzas se priorizaron por sobre las diferencias. Por otro lado, las revistas permitieron nuclear a los estudiantes, salir de sus casas de estudio y volverlos un interlocutor colectivo válido, conectándolos a redes vinculantes con otros actores que comenzaban a reaparecer públicamente durante este periodo (como ser los sindicatos y los partidos políticos). Rodríguez explica como estos clivajes políticos formaron parte de un subtexto que marcó culturas políticas más continuistas y rupturistas dentro de la universidad: 
no lo sentí tanto como un desencuentro intergeneracional, porque nos movíamos en la misma generación. Lo que había era que algunos estaban, si querés, por diversos motivos, más atados a la estructura que venía del pasado y otros estaban dispuestos a, con imaginación, dentro de una misma línea, generar cosas nuevas (...). Para la UJC era muy difícil concebir una estrategia diferente. Porqué el riesgo que recorrian era tan alto, que cualquier estrategia para ellos era exponerse. Entonces ahí empezamos a tener un choque natural entre visiones. Nosotros nos exponíamos, pero no era lo mismo que ser comunista (Rodríguez, J., comunicación personal, 9 de agosto de 2018).

Sin lugar a dudas estas diferencias tuvieron que ver con el surgimiento de sensibilidades generacionales distintas y tensiones entre visiones coexistentes; por un lado, aquellas que buscaron revindicar formas más culturales de participación como espacios de movilización y articulación alternativos utilizando los canales legales disponibles y, por el otro, aquellas que reivindicaron el retorno a formas de participación más orgánicas y lógicas políticas heredadas del viejo movimiento estudiantil.

La reconstitución del tejido social -democrático y plural-implicó apelar a nuevas formas de socialización propias de una identidad generacional en transiciónque se contrapuso a la identidad generacional de la década del sesenta y que intentó politizar- y muchas veces democratizar- nuevos espacios. Vemos como la nueva identidad del movimiento estudiantil que emerge en este contexto está plagada de tensiones intra-generacionales, que se expresaron a través de relaciones ambivalentes tanto en cuestiones organizacionales como en el discurso, y las revistas estudiantiles no estuvieron ajenas a estas tensiones, más bien fueron espacios para irlas resolviendo. El movimiento estudiantil que surge con la reapertura y que apuntó, por un lado, a la "reconstrucción de la memoria histórica del movimiento estudiantil y reivindicó el legado de la generación universitaria anterior a la intervención" (Markarian, Jung y Wschebor, 2009, p 4), por el otro, tuvo también que aggiornarse y apelar a una impronta generacional propia que le permitiera generar arraigo en las nuevas filas de estudiantes que ingresaban a la universidad. En la medida que el sentido de "lo político" debió resignificarse durante principios de los ochenta, esto implicó en sí mismo un quiebre en la sensibilidad estudiantil. "El tema de la innovación y la creatividad fue muy importante. La capacidad de salir de los canales normales y hacer cosas diferentes caracteriza la generación. Ante la imposibilidad, la creatividad de pensar otras cosas es muy importante" (Rodríguez, 2013). En algún sentido, y al igual que ocurrió en la región y en otros movimientos, el nuevo discurso político adquirió aspectos de índole más "posmodernos", caracterizados por una secularización de la práctica política y una revalorización del presente y la democracia en tanto fin en sí mismo.

Sin embargo, hay un afán constante por posicionar a la nueva generación de estudiantes dentro de una saga mayor que provee a los "recién llegados" de un arsenal de simbologías y banderas que son esenciales para una identidad en construcción. Como dice una nota en la revista Diálogo, "nuestra Universidad tiene una rica tradición estudiantil. Estudiantes han sido quienes le impulsaron a renovarse. Estudiante fueron quienes marcaron el rumbo en momentos de crisis. Estudiantes fueron también quiénes ofrecieron la mayor cuota de sacrificio y entrega. Todos los momentos claves de la vida nuestra universidad fueron testigos de este aporte estudiantil: cuando la universidad necesitó de ellos, los estudiantes no faltaron a la cita. Hoy, estamos viviendo uno de esos momentos" ("Democracia, autonomía y participación”, 1982, p. 4). Es decir, varios grupos de estudiantes comenzaron a verse a sí mismos como continuistas de una identidad colectiva de larga data, pero buscaron imprimirle un contenido nuevo, acorde 
a los tiempos que corrían. Las revistas permitieron comunicar esta nueva sensibilidad, a la vez que ayudaron a construirla. "Debemos aprender del pasado, pero no con nostalgia, con madurez" ("Democracia, autonomía y participación”, 1982, p.4). Dicha sensibilidad juvenil se entrelazó con nuevas ideas sobre la democracia, que la concibieron desde una acepción menos procedimental y más relacionada con lógicas de participación horizontal y de autogestión. Estas transformaciones estaban vinculadas, a su vez, con la circulación regional y global de nuevas prácticas y discursos culturales juveniles. La lucha por la democracia externa tuvo su correlato en las luchas por la democracia interna, con un marcado quiebre con formas de militancia de la izquierda tradicional.

Es sugerente un artículo que apareció en Diálogo en la sección "Para la Polémica”, titulado "Una generación sin vanguardia" que narra justamente esta crisis de identidad por la que atravesó la generación de la reapertura democrática que intentó encontrar en ese aparente vacío un terreno fértil para la emergencia de elementos nuevos. Dicha sección, buscó dar un espacio a colaboraciones que, sin expresar necesariamente la posición de la revista, "poseen innegables valores o especial interés, y a su vez abordan temas desde un punto de vista polémico o discutible" (Barone et al., 1982, p.42). A partir de una autocrítica, relacionada con la posición privilegiada de muchos estudiantes en relación con el conjunto de la estructura social, un creciente consumismo y una aparente apatía juvenil, se identifica una oportunidad para desarrollar una impronta generacional nueva, inédita, que permita devolver el rol protagónico a la juventud y a los estudiantes ("tenemos un mundo por delante, que sea nuestro") en contraposición al "medio castrador y tradicionalista". Sugerentemente se habla de "abrir nuevas puertas" a través de "nuevas llaves", pero para volver a ser, "vanguardia" (un concepto y una aspiración claramente heredadas de las generaciones anteriores) (Barone et al., 1982, p. 42). Esta tensión entre lo "nuevo" y lo "viejo", de un movimiento intentando reconstruirse hacia el futuro, pero una mirada en el pasado, se materializo en discusiones internas sobre el rol del movimiento estudiantil en democracia.

Es verdad que somos, "la generación del coletazo“, porque mal que bien tenemos reflejos de época, con más libertades (no con libertad), con algún concepto claro, que nos quedamos, nos dejaron, en cierta medida, sin raíces, sin enseñanzas, que nos limitan en la práctica, por sobre todo en eso.

Es así, que, dentro de este entorno, dentro de este medio castrador y, tradicionalista, intentamos crecer, vivir, aprender. Pero también creemos que esa castración fue y es no solo a nivel material, intelectual, sino fundamentalmente a nivel humano. (...)

Ahora bien, cabría la pregunta, ¿En qué grado no somos nosotros también culpables de esta situación? Pensamos que el sí; es contundente cuando miramos y nos desprendemos un poco de nuestras vivencias individuales, analizando más objetivamente nuestra generación y concluimos en lo apáticos, desmotivados y poco atractivos que somos los jóvenes en este país.

Vemos que la teoría nos rebasa, nos inunda, cuando vemos nuestra propia incoherencia al hablar de lo popular, al vestirnos y adornarnos con lo popular, al hablar del trabajador, de su realidad, y ver que pocos de nosotros han pisado una fábrica en su vida; es más, habla de la realidad del obrero y el papi lo sigue buscando, cuando el laburante cobra por mes apenas un salario que le permite subsistir; en cambio nosotros seguimos en la rosca consumista comprando vaqueros de medio palo o championes que valen cerca de 
uno, mientras que el trabajador la única marca de jeans que conoce es la del overol sanforizado por supuesto que viene de arriba, se lo da a la fábrica, porque para él son todos iguales. (...)

Somos "ilustres" porque hablamos de futuro y ni siquiera nos damos cuenta de que presente nos está tragando (...)

Es necesario siempre para abrir viejas puertas tener llaves nuevas, en este caso, nuevas formas aplicables para un país que vive muerto desde hace tiempo.

Hoy la responsabilidad de ser mejores nos corresponde solamente a nosotros, a la sangre viva de un pueblo, a los jóvenes. (...)

Hoy, aquí, ahora, tenemos cosas para decir, para hacer, cosas que importan, que valen, que son. Tenemos un joven y un mundo por delante, que sea nuestro.

¿Entonces como aceptar ser una generación innovadora de la nada?

¿Cómo aceptar pasar por la vida sin desafiarla, sin dejar huellas, sin descubrirnos?

¿Cómo dejar que la muerte nos viva y que irónicamente asista a nuestro propio entierro?

Tal vez la generación del silencio se hable algún día, como la generación muerta antes de nacer, o de aquella que, con muy poca vida, dio a luz un ser y fue vanguardia (Barone et al., 1982, p.43).

Este artículo surgió, junto con un aluvión de otras respuestas, como reacción a otro publicado en el número anterior, titulado "No somos vanguardia", donde se caracterizaba a la juventud de la siguiente forma: entraron a una secundaria intervenida, sólo conocieron los reglamentos que imponían los uniformes, las tarjetas de identificación los actos interminables que exaltaban un nacionalismo de extraña interpretación. Ni siquiera conocieron a los antiguos profesores, sólo los vacilantes y novatos sucesores, repitiendo un verso mal aprendido o simplemente inventado sobre moral, historia o política ("No somos vanguardia”, 1982, p. 33).

A partir de estos intercambios, es posible ver un afán por construir una esfera pública y repensar el lugar de los estudiantes dentro de la Universidad. Dicho esfuerzo implicó redefinir el lugar de los estudiantes desde una incorporación de elementos del pasado (reconociendo por ejemplo el legado de las generaciones estudiantiles anteriores) pero imprimiendo aspectos generacionales propios, vinculados a nociones de democracia mucho más participativas. Es significativo, por ello, que conceptos como el de vanguardia, fuertemente sugerentes para la generación estudiantil que irrumpió en el escenario nacional, regional e internacional en los 60 , volvieran a aparecer en otro contexto, pero teñidos de nociones que le eran ajenas a las viejas acepciones. Sin embargo, como dijimos, estas redefiniciones no se procesaron en todos los estudiantes por igual ni de las mismas maneras.

\section{Esa simbiosis entre el adentro y afuera}

Si se observa al movimiento estudiantil a través de los editoriales de sus revistas es posible ver indicios de una actitud paulatinamente más crítica y reivindicativa en relación con la Universidad, pero a través de las discusiones sobre la transición en la esfera universitaria y educativa, también se fueron gestando discusiones en torno a la transición y democracia en general (Serrentino, 1985). En tándem, y a medida que las revistas comienzan a exigir un rol más protagónico de los estudiantes para revertir la crisis institucional 
de la Universidad, demandando mayor participación en los procesos de reforma interna, y mientras la realidad del país avanzaba, la discusión sobre la participación interna comenzó a tener correlatos en disputas acerca del rol del estudiante en la transición política del país.

El trabajo del Foro Juvenil recoge este proceso de amplificación de las demandas y de constante paralelismo entre los embates autoritarios y los procesos de democratización que acontecían al interior de la universidad y los que ocurrían afuera. Por ejemplo, la nota de Trazo ("Requisitos para la transición”, mayo de 1982) al referir a los requisitos necesarios para una transición planteaba: "esto quiere decir que- hoy por hoy-el problema de la Universidad es el problema de la participación. Por lo tanto, y entendido el termino de "transición" como el pasaje de una situación dada a una futura (para nuestro caso, de una situación deficitaria actual a una situación ideal), esta habrá de entenderse en la Universidad como el pasaje de la no participación a la participación total, de la intervención a la autonomía" (Foro Juvenil, 1982, p. 12). Similarmente, en un editorial de Diálogo (junio 1982) titulado "La conformación de la incapacidad y la importancia", se plantea que "es la hora entonces, de que los universitarios reasumamos la responsabilidad de dirigir la Universidad. Es la hora de que los estudiantes, los docentes y los egresados discutamos y planeemos hacia adelante, porque la intervención extrauniversitaria se ha demostrado más que incapaz de hacerlo" (Foro Juvenil, 1982, p. 13). En Siembra (8 de junio 1982), en el artículo ¿Hacia dónde va la universidad?, y en referencia a la discusión en torno a una nueva Ley Orgánica para la universidad, se concluye: "consideramos que el destino de la Universidad no puede ser decidido solamente por un grupo de personas arbitrariamente designadas" (Foro Juvenil, 1985, p.12). En otro número de Trazo (9 de julio de 1982), en un artículo titulado ¿De qué transición me habla?, se llama precisamente la atención sobre ese juego entre la Universidad y el proceso político que atravesaba el país: "evidentemente un pasaje de una situación de hecho a una de derecho no se hace haciendo leyes, sino habilitando a quienes deben hacerlas en un marco de participación y libertades. Si no, no habrá consenso, y tampoco transición" (Foro Juvenil, 1985, p. 12).

El siguiente ejemplo de la revista Diálogo (de junio 1982), aunque extenso, es un ilustrativo fragmento que muestra este proceso de amplificación de las demandas y de identificación de las causas universitarias con las de la sociedad uruguaya en su conjunto (una suerte de "simbiosis" para usar las palabras de los propios estudiantes), con la concomitante ampliación de la noción de democracia. En este caso, vemos como, a través de un juego de espejos, se hace una crítica de la autoproclamada despolitización de la Universidad llevada adelante por la dictadura y se invoca la necesidad de repolitizar las problemáticas universitarias. La universidad debe ser vista, en esencia, como una institución política y como una institución democrática, y democrática en un sentido que ahora incluye la dimensión económica además de la participativa. En esta acepción de democracia, que para los estudiantes ha sido "tanto $y$ tan mal usada", se incluyen elementos de democracia económica que engloban, a su vez, la necesidad de revisar el carácter elitista de la Universidad y ampliar la participación social:

Hasta el año 1973 la Universidad de la República era en realidad la República de la Universidad. Vivíamos una Universidad politizada con signo opuesto al sentido democrático de la nación. En la universidad se discutian y profesaban idea foránea, contradictorias con nuestro estilo de vida. Podríamos seguir citando comentarios oficiales oficiosos, pero todos coincidentes. Desde hace años parece ser que todas las voces audibles están de acuerdo en esto, cuando de la universidad se habla. Es más, todo el mundo aparece convencido de que la supresión de esa realidad politizada era necesaria.... Por el contrario, 
como, en la versión oficial de intervención de la Universidad y de la Enseñanza en general, ha permitido la despolitización de la educación y la implantación del orden y la paz en los centros de estudio (...)

No es verdad que la universidad haya sido despolitizada y esto no por error en los métodos o en la elección de modelo sustitutivo. La verdadera causa consiste en que semejante propósito constituye una absoluta imposibilidad. La universidad es en esencia una institución política. (...)

Si proponemos una Universidad democrática y de ingreso libre, esta propuesta implica el concepto ideológico de la extensión de la educación y la participación social a las grandes mayorías de la población. Fácil es ver que esa opción universitaria trae aparejado una concepción sociopolítica, esto es, un modelo de sociedad democrática y abierta dentro de la cual esa universidad pueda funcionar y tener sentido.

La historia de nuestra universidad es el mejor ejemplo de esa simbiosis inevitable entre universidad y el país (...)

Queremos una universidad democrática porque entendemos que la misma no tiene sentido si no vuelca sus mayores esfuerzos al mejoramiento de la situación de las grandes mayorías olvidadas de la población, desarrollando en ese sentido, la permanente investigación en las ciencias y la cultura en general.

Queremos una universidad democrática para que puedan acceder directamente todas las clases sociales, multiplicando para eso los tipos de cursos y las carreras y atendiendo los horarios de quienes trabajan.
Queremos una universidad democrática y participante que se ocupe de buscar soluciones a los verdaderos problemas del país, realizando una labor fecunda y productiva, en lugar de anular aún más los lazos de dependencia de su desarrollo.

Queremos una universidad democrática que sea capaz de definir sus propios objetivos y los medios para lograrlo a través del cogobierno de docentes, egresados y estudiantes debidamente asociados, amparado por un auténtico estatuto de Autonomía. Éstos son apenas los lineamientos de los cuales emprendemos la tarea de repensar la Universidad. ("La Universidad es una institución política”, 1982, p.22-24).

Como suele ocurrir, la sinergia entre movimientos sociales y el contexto en que se desenvuelven no permite discernir claramente la direccionalidad y causalidad de su vínculo, pero es posible decir que los estudiantes modificaron su entorno, logrando articular cada vez más sus demandas con las de otros actores sociales y, a su vez, que la propia experiencia de participación y organización los fue modificando sobre la marcha. Es interesante ver como las revistas estudiantiles del 83 ya refieren a un movimiento estudiantil constituido que, aunque con discrepancias de rumbo y de métodos de lucha a la interna, se autoproclama como un todo unificado. Esta construcción de un discurso unificado, aglutinado en torno a una identidad común compartida, era condición sine qua non para pasar de la segunda a la tercera cara del poder e irrumpir en la escena pública con masividad y respaldo. El siguiente fragmento del editorial de la revista Diálogo de marzo de 1983 ejemplifica esto:

En el ámbito estudiantil existe un acuerdo más o menos expreso en cuanto a las grandes definiciones de la Universidad que queremos. La concebimos democrática en su estructura interna 
y democratizadora en su función social y pensamos volcada a la búsqueda de un auténtico desarrollo social y económico, que nos libere de la pobreza y la dependencia. Lo que no siempre estamos tan de acuerdo, es en los medios para buscar esos fines.

De esas discrepancias en cuanto a medios, están compuestos nuestros más grandes errores y fracasos.

Puede afirmarse que los objetivos elevados y positivos en lo que estamos de acuerdo para la Universidad, se torna inalcanzable si no somos capaces de unificar nuestro accionar y empujar todos en una misma dirección.

Si queremos ser una fuerza influyente para el futuro de la universidad, debemos aprender a actuar como un verdadero grupo de presión; demostrar con nuestros actos y la coherencia de nuestras posiciones, no solo que somos un sector capaz de cogobernar la universidad, sino que sin nuestra participación sería imposible encontrar una salida verdadera y durable ("¿De quién depende el futuro?", 1983, p. 3).

La revalorización de a la democracia, que formó parte de un proceso más general que se evidenció en el discurso de otros actores de izquierda en Uruguay y la región, adquirió en el caso de los estudiantes significados complejos que distaron de definiciones meramente procedimentales de la democracia. Al llegar a 1983 es posible hablar de una identidad colectiva común que se constituyó en franca oposición a ese "otro" que era la dictadura (con sus múltiples caras, tanto al interior como al exterior de la Universidad). Las revistas son un testimonio de esa búsqueda que se dio dentro del estudiantado durante la transición a la democracia por el significado de ser "estudiante”, “joven” y "movimiento estudiantil” y, más allá de las pugnas por los significados, sus significados se contrastaron con los que le había dado el autoritarismo. El proyecto político del autoritarismo en la universidad buscó despojar a la universidad de la "vieja" política; la (re)politización de la vida universitaria, a través de instrumentos como las revistas, fue una de las primeras batallas ganadas a la dictadura.

\section{Conclusiones}

"Estudiante, sal afuera / venciendo la soledad / la noche se hace día / sal afuera y lo verás. / Nuestra casa destruida / el pueblo levantará / de esa pared solidaria/ somos un ladrillo más" (Faroppa y Espasandín, 1983).

Las revistas estudiantiles que pulularon durante los primeros años de la transición a la democracia en Uruguay en todas las facultades de la Universidad de la República fueron un elemento fundamental en la reconstrucción del movimiento estudiantil como movimiento social masivo y visible. En tanto catalizadoras de una sensibilidad juvenil en construcción, las revistas se constituyeron en medios alternativos de comunicación que permitieron generar espacios contrahegemónicos de socialización que supieron jugar un rol central tanto internamente como externamente. Internamente, las revistas se impulsaron a través de espacios de organización que permitieron a los estudiantes relacionarse en términos que ellos mismos vieron y sintieron como más democráticos, horizontales y participativos y que permitieron ir generando mecanismos de socialización política y sentar las bases para una futura militancia gremial. Externamente, las revistas trascendieron ampliamente los ámbitos meramente universitarios y se constituyeron en medios de comunicación alternativos a los medios oficialistas, dando voz a este actor social.

Las revistas estudiantiles fueron transitando un proceso de ampliación de sus demandas, de temas meramente universitarios particularistas a temas neta y 
abiertamente políticos. Dentro de este proceso, se dio una revalorización de la democracia (entendida ahora en sus dimensiones sociales, culturales y económicas) como fin en sí mismo y, concomitantemente, estas visiones ampliadas de la democracia se relacionaron sensibilidades y códigos generacionales que difirieron a los de sus antecesoras. Las conversaciones y discusiones sobre la transición y la democracia en la esfera universitaria y educativa tuvieron constantemente sus correlatos en discusiones acerca de la transición y democracia en general y, a medida que los números sorteaban la censura, se radicalizaban en sus planteos.

Aunque la participación de los ciertos sectores del estudiantado en las movilizaciones por el "NO" en el plebiscito del ochenta dieron cuenta de un proceso paulatino de reconstitución del movimiento estudiantil universitario, es recién durante el año 1983, entre el 1 de mayo y la organización de la Semana del Estudiante (con las movilizaciones que se generan en torno a dicho evento), que se puede hablar de una capacidad de movilización masiva y un ejercicio real de la capacidad de incidencia del movimiento estudiantil uruguayo de la transición. El 1983 eclosionó el potencial de resistencia acumulado hasta el momento y apareció un plan de lucha y movilización más "radical" que el que había prevalecido hasta el momento. Sin embargo, para que esta primera cara del poder estudiantil quedara de manifiesto, fue necesario primero dar visibilidad a las necesidades y demandas estudiantiles, conectarlas con las problemáticas que acontecían más allá de los muros universitarios, y lograr que los estudiantes dejaran de percibirse como actores aislados para visualizarse como agentes activos de un movimiento social constituido (es decir, moverse desde los intersticios de la tercera y segunda cara del poder a la primera). Sin estos pasos previos, no sería posible hablar de generación del 83.

La "Marcha del Estudiante" creada para la Semana del Estudiante e interpretada por diversas murgas estudiantiles de varias facultades buscó dar cuenta de ese aparente "despertar" del movimiento estudiantil. Un movimiento estudiantil que se vio a sí mismo como un "ladrillo más" de un pueblo "que se levantaba". Se produjo un cambio fundamental entre 1980 y 1983; estudiantes como Juan, huérfanos "de memoria histórica y de elementos para comprender su presente”, había logrado reconstruir un legado común y pasar a sentirse parte de un colectivo mayor, "salir afuera" e intentar reconstruir esa "destruida casa" que era la Universidad (Marcha del Estudiante, 1983). Una vez transitados y procesadas las tensiones inherentes a esos intersticios, las revistas dejaron de jugar un papel central y las voces comenzaron a ser expresadas y escuchadas de otras formas. Sin embargo, una vez instaurada la democracia, con el proceso de normalización de la universidad y las elecciones universitarias, los clivajes ideológicos que estaban latentes volvieron a emerger y adquirir preponderancia dentro de las identidades estudiantiles.

* Investigación realizada en el marco de Maestría en Historia Rioplatense, Facultad de Humanidades y Ciencias de la Educación (UDELAR) y del proyecto "Las disputas en torno a la idea de democracia: debates, institucionalidad y prácticas políticas, sociales y culturales durante la transición uruguaya (1980-1995)" a cargo de Aldo Marchesi y Diego Sempol, GEIPAR, CSIC. Estoy muy agradecida por los comentarios recibidos por parte del grupo del proyecto de investigación, por parte del profesor Demasi y los árbitros anónimos que evaluaron este trabajo.

\section{Referencias}

Araujo, A. M., Del Singore, G., Muñoz, C. B., y Rodríguez Larreta, J. (1991). Jóvenes: una sensibilidad buscada. Montevideo, Uruguay: Nordan Comunidad.

Bachrach, P., y Baratz, M. S. (1962). The Two Faces of Power. American Political Science Review, 56, 941-952. 
Barone, G, Romano, F., y R. Duce. (1982). Para la Polémica: Una generación sin vanguardia. Revista Diálogo, 1(5), 42-43. Recuperado del Archivo General de la Universidad de la República.

Bayce, R. (1989). Cultura política uruguaya: desde Batlle hasta 1988. Montevideo, Uruguay: Fondo de Cultura Universitaria.

Bresnahan, R. (2007). Community radio and social activism in Chile 1990-2007: Challenges for grass roots voices during the transition to democracy. Journal of Radio Studies, 14(2), 212-233.

Breve reseña. (1984). Boletín Centro de Estudiantes de Ingeniería, 2(15), p. 1. Recuperado del Archivo General de la Universidad de la República.

Buchanan, P. G. (1997). Counterhegemonic strategies in neoliberal Argentina. Latin American Perspectives, 24(6), 113-132.

Caetano, G., y Rilla, J. (1987). Breve Historia de la Dictadura. Montevideo, Uruguay: Centro Latinoamericano de Economía Humana.

Caetano, G., y Rilla, J. (1995). La izquierda uruguaya: tradición, innovación y política. Montevideo, Uruguay: Trilce.

Calicchio, L. (2002). El abandono del activismo universitario hacia fines de la década del ochenta. Notas para una historia de la ASCEEP FEUU (Tesis de Maestría en Ciencias Sociales). Facultad de Ciencias Sociales, Universidad de la República, Montevideo, Uruguay.

Dahl, R. A. (1957). The concept of power. Behavioral Science, 2(3), 201-215. https://doi.org/10.1002/bs.3830020303

De Giorgi, A., y Demasi, C. (coords.). (2015). El Retorno a la Democracia: Otras miradas. Montevideo, Uruguay: Fin de Siglo/ Universidad de la República.

Demasi, C. (2005). La "apertura democrática» como tema de análisis. Revista La Gaceta.

Democracia, autonomía y participación. (1982, marzo). Revista Diálogo, 1(3), 4-5. Recuperado del Archivo General de la Universidad de la República.

¿De quién depende el futuro? (1983, marzo). Revista Diálogo, 2(10), p. 3. Recuperado del Archivo General de la Universidad de la República.
Diálogo: una experiencia de participación. (1982, octubre). Ponencia presentada en Simposio Los jóvenes y los medios de comunicación, Foro Juvenil, AMPU, Montevideo. Recuperado del Archivo General de la Universidad de la República.

Editorial. (1981, noviembre). Revista Encuentros Veterinarios, 1(1), p.1. Recuperado de Colección privada Pablo Guyer.

Editorial. (1983, julio). Revista Encuentros Veterinarios, 3, p. 1. Recuperado de Colección privada Pablo Guyer.

El estado actual de nuestras calles. (junio, 1982). Revista Diálogo, 1(5), p. 13. Recuperado del Archivo General de la Universidad de la República.

Escobar, A., y Alvarez, S. E. (1992). The Making of Social Movements in Latin America: Identity, Strategy and Democracy. Boulder, C0: Westview.

Faroppa, J., Espasandín, M. (1983). Marca del Estudiante [canción]. Recuperado el 7 de noviembre de 2018 de https:// www.ivoox.com/marcha-del-estudiante-asceep-feuu-audios-mp3_rf_266964_1.html

Filgueira, C. (1985). Movimientos Sociales en la restauración del orden democrático: Uruguay, 1985. En C. H. Filgueira (Comp.), Movimientos Sociales en el Uruguay de hoy (pp. 09-50). Montevideo, Uruguay: CLACSO/CIESU/Ediciones Banda Oriental.

Foro Juvenil. (1985). Movimiento Estudiantil y Transición Universitaria (Trabajo en base a servicios de documentación propios). Departamento de Estudiantes del Foro Juvenil, coordinado por José Felipe Serrentino.

Foro Juvenil. (1982, octubre). Simposio Sobre Jóvenes y los medios de comunicación. AMPU. 1-3 de octubre, 1982. Recuperado del Archivo General de la Universidad, Carpeta G83203.

Garretón, M. A. (1989). La posibilidad democrática en Chile. Santiago de Chile, Chile: FLACSO.

González, L. E. (1985). Transición y restauración democrática. Montevideo, Uruguay: CIESU.

González Vaillant, G. (2015). Building Counter-Power: A comparative study of student movements in Argentina and in Chile at the turn of the 21st century (Tesis de Doctorado). State University of New York en Stony Brook. 
Juan y su generación. (setiembre, 1982). Revista Diálogo, 1(8), 3435. Recuperado del Archivo General de la Universidad de la República.

Jung, M. E. (2010). La reorganización del movimiento estudiantil y la restauración democrática en la UDELAR. 1980-1983. Encuentros Uruguayos, 4(4).

La Juventud universitaria y los medios de comunicación. (1982, agosto). Revista Causa, 1(2), 17-18. Recuperado del Archivo General de la Universidad de la República.

La Universidad es una institución política. (1982, junio). Revista Diálogo, 1(5), 22-24. Recuperado del Archivo General de la Universidad de la República.

Lugo-Ocando, J. (2008). An introduction to the maquilas of power: media and political transition in Latin America. En J. Lugo-Ocando (Ed.), The media in Latin America (pp. 1-12). Berkshire, Inglaterra: Open University Press.

Luke, S. (2005). Power: A Radical View (2 ${ }^{\text {nd }}$ expanded edition). Londres, Inglaterra: Macmillan. (Trabajo publicado originalmente en 1976)

Marchesi, A. (2009). "Una parte del pueblo uruguayo feliz, contento, alegre". Los caminos culturales de consenso autoritario durante la dictadura. En C. Demasi, A. Marchesi, V. Markarian, A. Rico, y J. Yaffé, La dictadura cívido militar. Uruguay 1973-1985 (pp. 337-398). Montevideo, Uruguay: Banda Oriental.

Markarian, V., Jung, M. E., y Wschebor, I. (2008). 1983: La generación de la primavera democrática. Colección Aniversarios Universidad de la Republica 1983-2008, volumen 5. Montevideo, Uruguay: Universidad de la República.

Mieres, P., y Argones, N. (1989). La polémica en el Frente Amplio: ¿Pugna por contenidos organizacionales o institucionales? Cuadernos del CLAEH, 49.
No somos vanguardia. (1982, abril/mayo). Revista Diálogo, 1(4), p. 33. Recuperado del Archivo General de la Universidad de la República.

Perelli, C. y Rial, J. (1986). De Mitos y Memorias Políticas. Montevideo, Uruguay: Ediciones de la Banda Oriental.

Porrini, R. (2012). A veintinueve años de la marcha del estudiante de 1983: Obreros y estudiantes. Trabajo \& Utopía (órgano de difusión del PIT-CNT elaborado por el Instituto Cuesta-Duarte).

Rilla, J. (1997). URUGUAY 1980: Transición y democracia plebiscitaria. Nueva Sociedad, 150, 77-83.

Rodríguez, J. (2013). Estudiantes y jóvenes generaciones de la FEUU a la ASCEEP-FEUU. Presentado en Jornadas a 40 años del Golpe de Estado y Dictadura, Mesa 12. Universidad de la República, Montevideo, Uruguay.

Sempol, D. (2004). Los "mártires" de ayer, los "muertos" de hoy: El movimiento estudiantil y el 14 de agosto 1968-2001. En A. Marchesi, V. Markarian, Á. Rico y J. Yaffé (Eds.), El presente de la dictadura: Estudios y reflexiones a 30 años del golpe de Estado en Uruguay (pp. 169-183). Montevideo, Uruguay: Trilce.

Serrentino, J. F. (Coord.). (1985). Movimiento Estudiantil y Transición Universitaria. Foro Juvenil. Trabajo en base a servicios de documentación propios.

Una causa que es nuestra. (1982). Revista Causa, 1(1), p. 1. Recuperado del Archivo General de la Universidad de la República.

Una Revista Joven. (1982, abril). Diario La Democracia, p. 5.

Wiley, S. B. C. (2006). Assembled agency: Media and hegemony in the Chilean transition to civilian rule. Media, Culture and Society, 28(5), 671-693. 